\title{
Problems of Defining ANd SubSequently Measuring SURFACE ROUGHNESS IN APERIODIC SURFACES
}

\author{
Dana Kubátová
}
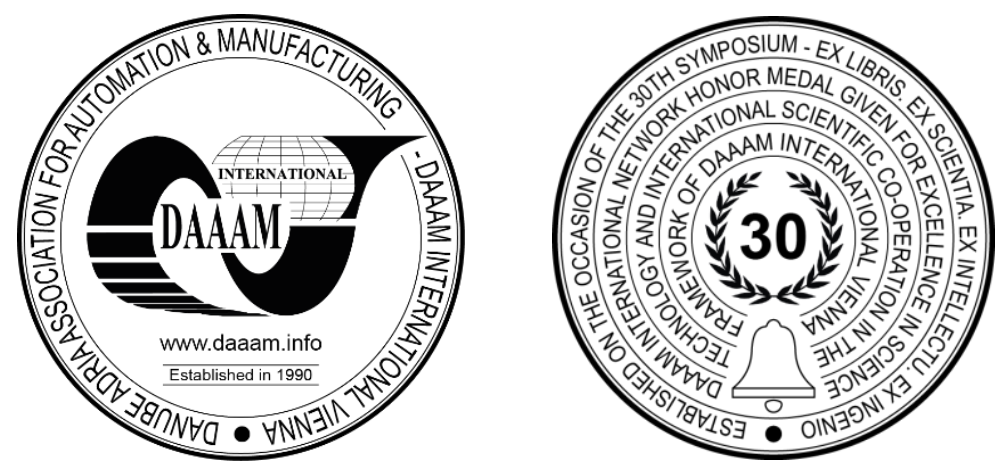

This Publication has to be referred as: Kubatova, D[ana] (2020). Problems of Defining and Subsequently Measuring Surface Roughness in Aperiodic Surfaces, Proceedings of the 31st DAAAM International Symposium, pp.0236-0243, B. Katalinic (Ed.), Published by DAAAM International, ISBN 978-3-902734-29-7, ISSN 1726-9679, Vienna, Austria DOI: $10.2507 / 31$ st.daaam.proceedings.033

\begin{abstract}
The concept of surface roughness plays an irreplaceable role in technical practice. It has been evaluated by various techniques and various mathematical procedures since the 1920s. However, the evaluation is often underestimated and reduced to finding the parameters $\mathrm{Ra}$ or $\mathrm{Rz}$. This is done in the same way for both periodic and aperiodic surfaces. However, this is a completely unacceptable simplification of the issue, and the following article sheds light on a "small" part of the extensive issue. The aim of the article is to point out the difference between measuring the surface roughness of periodic surfaces (the technical practice for the most common surfaces) and non-periodic surfaces. The article describes contact and non-contact systems of surface roughness measurement for periodic and aperiodic surfaces and tries to lead the reader to think about the accuracy of the procedure for measuring the surface.
\end{abstract}

Keywords: Surface roughness; Periodic surface; Aperiodic surface; Contact measurement; Non-contact measurement.

\section{Introduction}

The development of measuring and evaluating surface structures has seen significant qualitative technical progress in recent years. Leading manufacturers of measuring technology have actively responded to the new requirements for the quality of functional surfaces of components made of advanced construction materials combined with high accuracy of dimensions, shape, and mutual position of often small functional surfaces. The development of measuring technology is significantly influenced by the technical needs of users and their economic capabilities. Given that single-purpose measuring devices are used to check surface structures; it is clear that manufacturers are involved in increasing the quality of their devices. [2] The result of this comprehensive development is not only an increase in the technical level of existing methods for measuring and evaluating surface structures but also the preparation of new methodologies, measuring systems, and systems for assessing and evaluating the surface structure. But despite this progress, in practice, we still encounter the opinion that the evaluation of surface integrity is unnecessary and does not add any further knowledge to the potential for comprehensive optimization of the production of a given part or component.

The surface properties can significantly affect the mechanical, electrical, and chemical behavior of a measured part. The roughness of a surface affects its quality to a large extent. Characterizing surfaces in terms of roughness is technically very important because the evaluation of roughness offers opportunities for further optimization. [3] Many industries, for example medicine, automobile, aerospace, and electronics, benefit from evaluating the surface using roughness measuring devices (roughness gauges). 
The aim of this article is to map the emerging surface structures and clearly define the procedure for setting and measuring the surface for each type of surface (periodic surface or aperiodic surface). This article is written mainly due to the unification of the measurement procedure among practitioner's people. The goal of this article is to appraise to reader's information about the problem of the procedure of measuring different surfaces. [7]When the information about the procedure of measuring will know a lot of peoples, it will be easier to get the same results from measure process and achieve the same result regardless of what equipment and in how equipped the metrological laboratory the measurement will take place.

The outputs of this article will then be incorporated into a comprehensive methodology for selecting a software filter for measuring surface roughness. This methodology is created within the Regional Institute of Technology at the University of West Bohemia in Pilsen. The methodology aims to facilitate the work of man in evaluating the surface roughness. And this article is one of the supporting points for this methodology.

First it is necessary to say what surface roughness is. The basic definition according to EN ISO 4287 (describing terms, definitions, and surface parameters) stipulates that roughness is the "sum of surface irregularities with a relatively small distance, which inevitably arises during production or its influence" (Fig. 1).

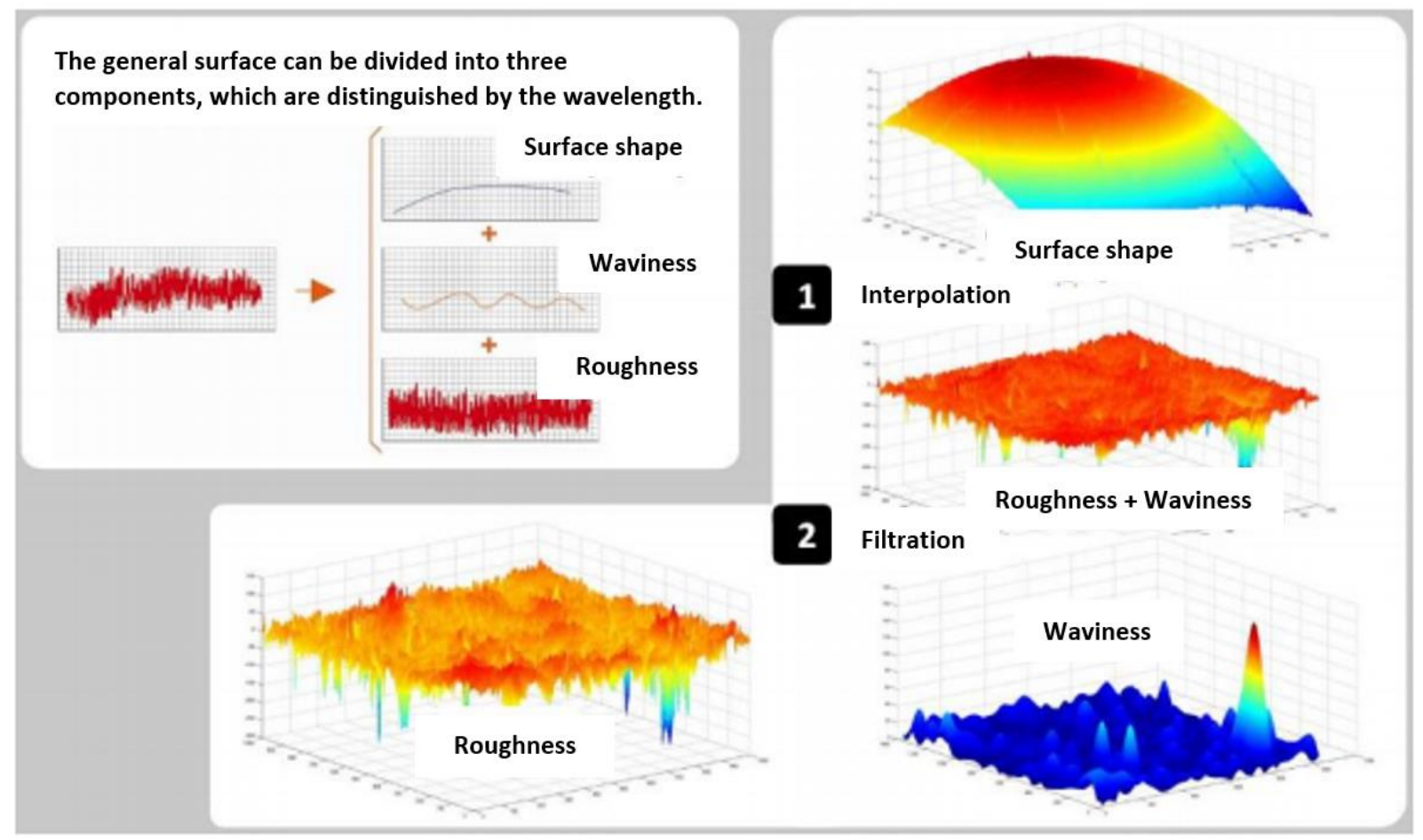

Fig. 1. Surface composition [10]

If we look at a real surface, we can find micro-roughness (which is the surface roughness caused by the traces left by a cutting tool or abrasive) and macro-roughness (which is called surface waviness and is most often caused by vibrations of the Machine - Tool - Work piece - Environment system). In the case of surface shape, these irregularities overlap and must be filtered out (i.e. divided into surface roughness and waviness).

The functional surfaces of the parts are usually finished by chip or chip-less machining. The designer prescribes the basic properties of the surface with respect to its intended function. The task of technology is to implement this regulation. In the ideal case of completely flawless production, the finished part is directly ready for functional use. In common practice, however, the conditions of the production process do not remain completely unchanged, and therefore it is necessary to include control operations to detect all deviations that may affect the function of the part (e.g. deviations in dimensions, shape, surface quality, the hardness of part material, etc.). Changes in the production process affect the surface structure in various ways, e.g. the effect of tool wear, the stress in the material, the use of unsuitable cutting conditions, etc. [3], [4] It is important that measuring the surface structure not only determines the actual surface condition but at the same time, it also provides data for the regulation of the production process and the prediction of the functional properties of the surface.

The surface structure that is created during machining depends in most cases on the machining method used. Depending on the method, two types of marks may appear on the surface. The direction of irregularities on the surface can be either distinct (periodic - fig. 2) or indistinct (aperiodic - fig. 2). A periodic surface is created during turning, milling, drilling operations, etc. It is caused when a tool creates grooves on the surface of the part in a certain direction. An aperiodic surface is created during casting, forging, etc. Somewhere on the border are finishing methods such as grinding, lapping, or polishing, which at some point create both types of surfaces on the work piece. 

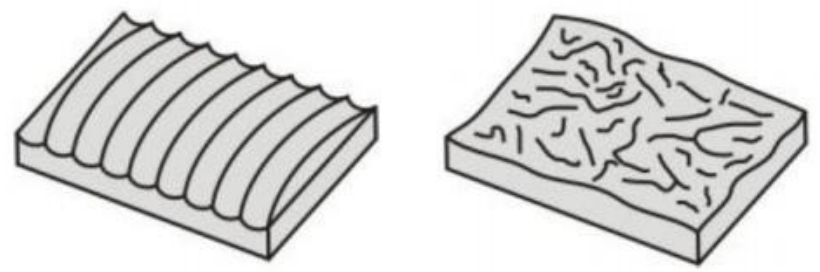

Fig. 2. Periodic and aperiodic surface

The periodic surface is a surface with a significant direction of unevenness. In order to measure meaningful values, it is necessary to observe the scanning direction of this surface perpendicular to the direction of the unevenness. Without this, the sensor would measure smaller values than the actual ones when moving in the direction of the unevenness, and the surface would appear more accurate at first glance.

The aperiodic surface is a surface with an indistinct direction of unevenness. Unlike a periodic surface, the scanning direction is not important here, because the inequalities are random and disordered. With this type of surface, it is always necessary to perform several measurements in different directions to find the maximum value of a particular surface roughness parameter. [5]

\section{Surface roughness measurement}

Two basic methods for measuring surface roughness are currently used in technical practice.

1) Qualitative methods (comparing a sample card with a real surface) - surface sample cards are still used today, or comparative microscopes. Here, however, it should be noted that these are fairly outdated methods, based on the individual abilities of the surface assessor (Fig.3). [6]
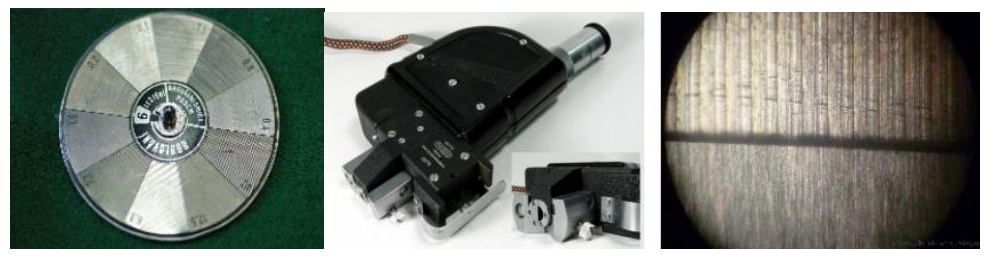

Fig. 3. Components for roughness measurement - comparing a sample card with a real surface

2) Quantitative methods (parametric, using a mathematical description of surface parameters) - in today's practice profilometers (Fig. 4) are commonly used. They are often called 'roughness gauges', which is not entirely correct because in addition to roughness these devices can also measure waviness and often shape. There are both contact and non-contact roughness gauges. [6]

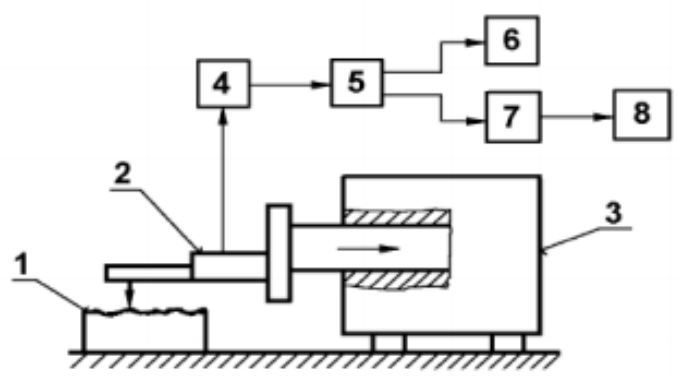

1. measured part

2. sensor head with measuring tip

3. feed mechanism

4. amplifier

5. filter

6. registration unit

7. signal processing unit

8. display

Fig. 4. Diagram of a touch device for measuring roughness

\subsection{Measurement of an aperiodic surface using contact method}

This is the most widely used surface evaluation method, which measures the surface profile using high-sensitivity instruments. A contact device has a specially modified tip that scans the coordinates of a surface, which are then computerprocessed. This is one of the oldest evaluation methods, and has been used since the 1920s. 
The movement of the probe must be very precise in terms of straightness and uniformity. The speed must be chosen with regard to the dynamic properties of the sensing system. The vertical movement of the tip as it passes across the protrusions and depressions is converted into an electrical signal by an inductive meter. This is then further computerized and subsequently evaluated. This sensing system is characterized by a small measuring force (i.e. a small force pressing the tip against the surface), which minimizes the risk of damage to the measured surface. [8], [9]

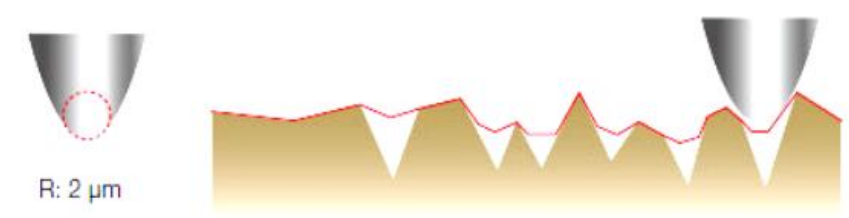

Fig. 5. Measurement of surface roughness by the contact method [3]

As can be seen from the description above, the type of surface on the tested part is important when selecting the system for measuring the surface. Apart from the radius of curvature of the probe $(2 \mu \mathrm{m}, 5 \mu \mathrm{m}, 10 \mu \mathrm{m})$, the following parameters are used in the measurement: the angle of the tip of the probe $\left(60^{\circ}, 90^{\circ}\right)$; measuring pressure force (approx. $\left.0.00075 \mathrm{~N}\right)$, and the rate of change of the measuring force. See article XYZ (soft filter selection methodology). The direction of scanning the primary profile, from which the resulting values of roughness, corrugation, or profile shape are subsequently processed, is also important for the contact measurement method (Fig.5). The scanning direction should always be perpendicular to the texture of the unevenness on the surface of the part, which is very difficult to find for an aperiodic surface. It is always necessary to make several measurements in different directions to ensure the measurement of the real maximum roughness value on the inspected surface.

\subsubsection{Instrument settings for measuring aperiodic surfaces}

Setting the device for contact measurement must be done in several steps. In the first step, one of the selected surface roughness parameters $(\mathrm{Ra}, \mathrm{Rz})$ is estimated visually using surface sample books (Fig. 6). Then, according to the estimated value of roughness, the basic evaluated and measured length is selected from the tables in the standard EN ISO 4287, then the size of the tip is selected for the measurement. A test measurement is made with the set estimated values, and according to the results of this, either the values (cut-off, measured length, evaluated length) are further adjusted or a real measurement is performed.

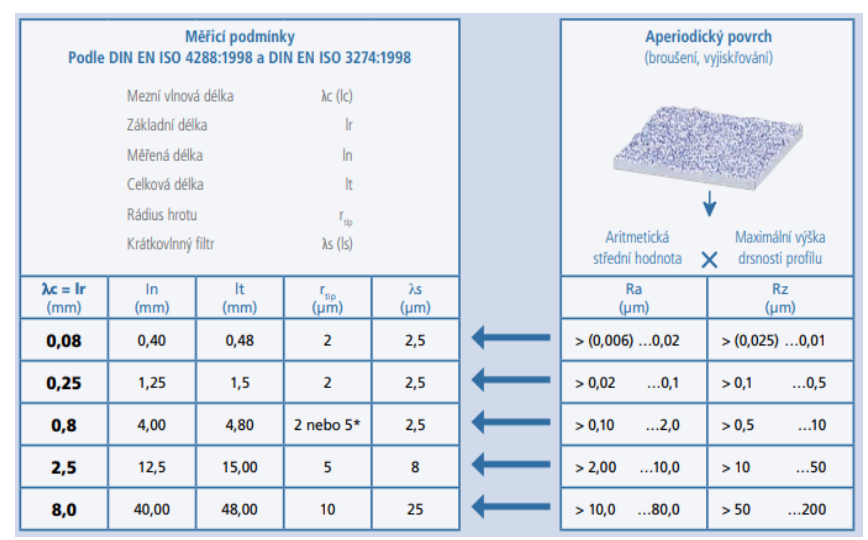

Fig. 6. Table for setting the device before measuring [4]

\subsection{Measurement by non-contact method}

The principle of the non-contact method (see Fig. 7) is based on the use of a light beam, which is projected on to a rough surface. The irregularities on the inspected surface scatter the light beam. With an ideally smooth surface, there is no light scattering, and the beam is reflected directly back to the source. The greater the roughness, the lower the mirror component, and the higher the scattering components. If the height of the irregularities is less than the wavelength of the scattered light, diffraction vectors and the scalar Beckmann theory are used to determine the surface roughness. When it is difficult to obtain the analytical value of the roughness from light scattering, Gaussian normal distribution is used for parameters to obtain precise electromagnetic boundary conditions. 


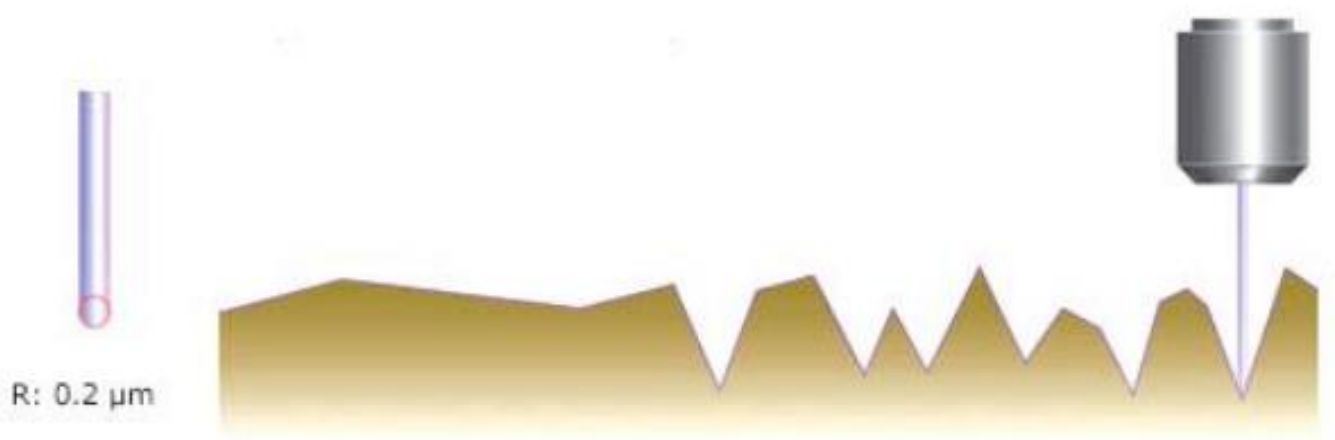

Fig. 7. Measurement of surface roughness by the contactless method [3]

A great advantage of non-contact surface roughness measurement is the possibility of measuring both surface roughness and profile surface measurement. This allows the standard EN ISO 4287 to be used for non-contact evaluation of surface roughness. The machine is set before measuring according to the tables in the standard. The setting and evaluation of the measured length and the value of the cut-off filter is based on the estimated values of the parameters Ra and Rz. Information for this setting is taken from standard EN ISO 25 178. A test scan is performed in the next step. If the estimate is incorrect (the image of the area on the PC has gaps or has areas without displayed roughness, it is necessary to change the settings and perform the test scan again. If the scan meets our requirements, an image of the measured surface is displayed.

It is usually necessary to continue processing the image so that the surface can be evaluated. First, it is necessary to align the surface. After alignment, it is necessary to intersect the measured surface with a plane so that the results are not distorted, because the device reads the values from its virtual plane, and therefore it is necessary to define the plane.

After this operation, a cut is made perpendicular to the tool marks on the measured surface. The cut can be made in two basic ways. The first variant is mostly used for periodic surfaces and is guided by a straight line perpendicular to the marks on the surface. (Fig. 8) The second variant uses the so-called 'ZIK-ZAK' cut. (Fig. 9) This cutting method is mainly used for aperiodic surfaces and allows us to find the maximum values for the selected parameters. Another use of this cut is when we have scanned a smaller (shorter) area than the required length for a straight cut. After this step, the resulting parameter values, including a graph of the profile, are displayed on the monitor. Now it is up to the operator to decide what they want to evaluate, and the given values will be displayed accordingly.

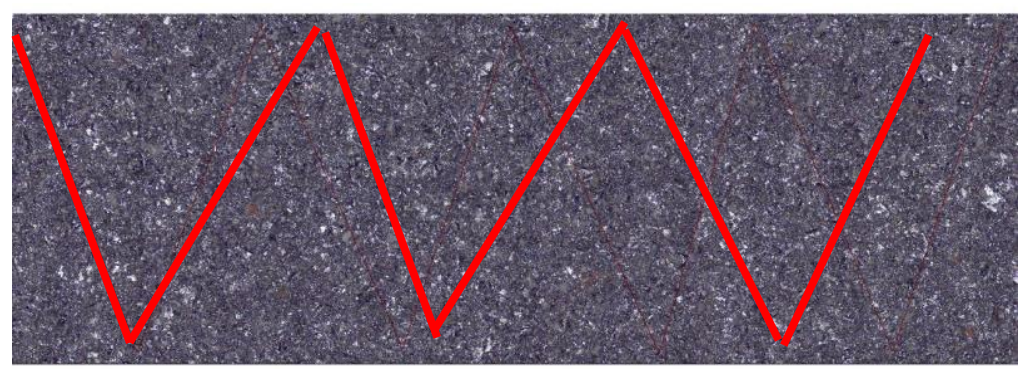

Fig. 8. Zik-Zak measuring method for roughness measuring

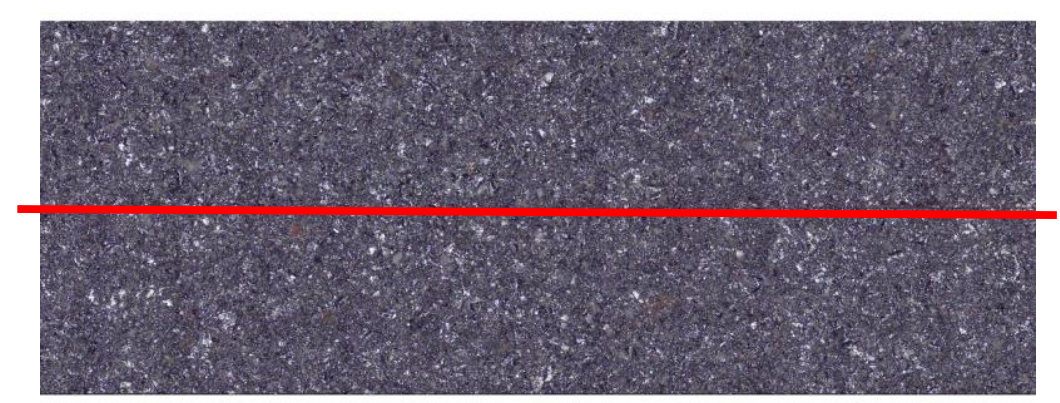

Fig. 9. Line measuring method for roughness measuring 


\section{Roughness parameters for evaluated a surface}

When a measured profile has been obtained, a second problem occurs, namely the choice of which parameters are evaluated. At present, it is possible to measure an inexhaustible number of parameters, whether height parameters according to the EN ISO 4287 standard ( $\mathrm{Ra}, \mathrm{Rz}, \mathrm{Rv}$ or Rp, etc.), shape parameters according to the same standard (Rdc, $\mathrm{Rmr}$ (c), etc.), Motif method parameters according to the standard EN ISO 12085 (R, W, Rx, etc.) up to the parameters of the load-bearing part according to the standards EN ISO 13565-2 and 13565-3 (Rvk, Rpk, etc.). Each of the parameters has its justification for being included in the evaluation of surface roughness. It is up to the surface evaluator to choose how a parameter will be evaluated, based on how the inspected part will be used.

The most frequently used and least informative parameter is the parameter Ra - Mean arithmetic deviation of the profile. This characteristic does not indicate what the surface looks like, it evaluates the same value of Ra for different profile shapes, but it is quite a cheap means of checking, for example, tool wear during machining. (See Fig.10)

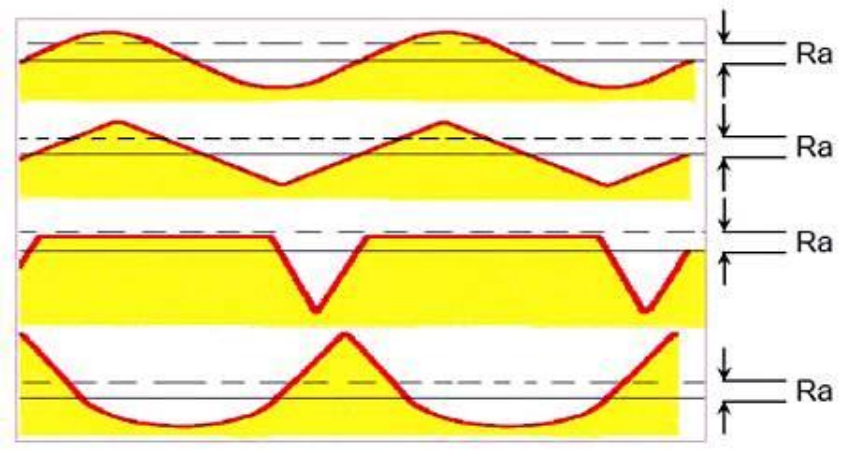

Fig. 10. Differences between surface structure and the value of parameter Ra [5]

The average square deviation of the profile $\mathrm{Rq}$ - As a rule, it shows higher values than $\mathrm{Ra}$, because $\mathrm{Rq}$ is more sensitive to unwanted depressions and protrusions of the investigated surface. But even for more significant values, this parameter is not so much used in general engineering. The main use is mainly in the optical industry. [8], [12]

Skewness Rsk - Indicates the displacement of the density function from the Gaussian normal distribution. In terms of profile shape, it better distinguishes surfaces with the same Ra values. When the peak of this function is shifted towards the depressions of the unevenness, the surface has more articulated protrusions and they occur in a larger number. The function with the apex shifted towards the protrusions shows larger and deeper depressions on the surface (See Fig.11). [1], [12]

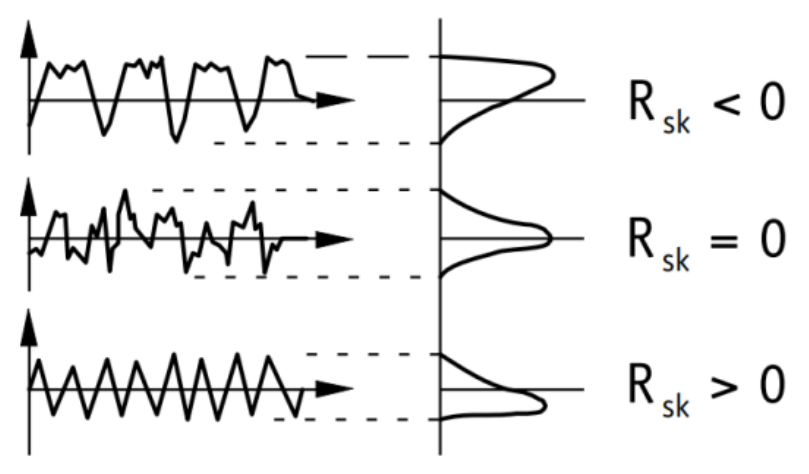

Fig. 11. Parameter Rsk [12]

The highest point of the profile $\mathrm{Rz}$ - This height parameter is significant especially for highly stressed components due to the large distance between the depressions and protrusions, which tend to crack. It has an unstable and variable value and is also affected by protrusions and grooves of the surface profile. [12], [11]

Parameters of the Abbot curve - parameters of load-bearing parts - Rvk, Rpk, Rk, Mr1, Mr2 - the shape of the curve is characteristic for different finishing methods, it is especially important for the assessment of loaded functional surfaces, solving problems of friction, lubrication and wear. In practice, the curve is replaced by straight lines, then the upper part (Rpk) corresponds to the surface protrusions, the middle part (Rk) corresponds to the profile core and the lower part of the curve (Rvk) describes the size and frequency of depressions on the surface (Fig.12 and 13). [1], [12] 


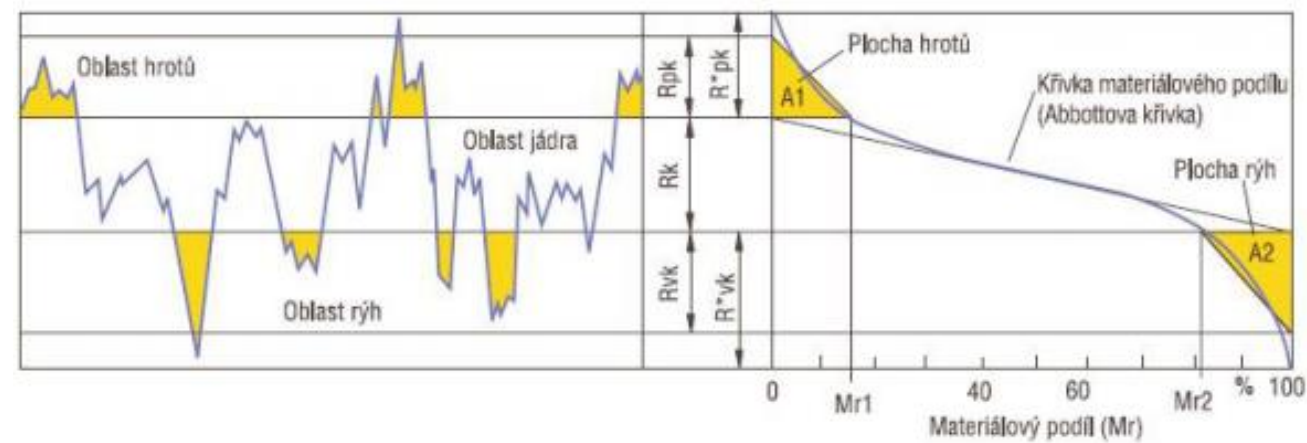

Fig. 12. Roughness profile and Abbott curve [5]

The small value Rk (small slope of the middle part of the support curve) corresponds to fine finishing operations (e.g. lapping). As a loaded contact surface, it slowly wears out, i.e. there are small changes in specific pressure. All this indicates practically unchanged contact (functional) conditions. The smaller the depth of the core, the flatter the character of the surface. The greater the depth of the core, with gradual wear, corresponds to a more pronounced change in specific pressure. In this case, the wear of the contact surfaces takes place faster, and only in the lower part of the depth of the profile core is a higher load-bearing proportion of the surface profile achieved. [12]

\begin{tabular}{|c|c|c|c|c|}
\hline Profily & $\mathbf{R}_{\text {. }}$ & $\mathbf{R}_{\mathrm{a}}$ & Mrese.25 & Abbottova krilvka \\
\hline$\sqrt{ } \cap$ & 1 & 0,25 & $75 \%$ & \\
\hline & 1 & 0,25 & $15 \%$ & \\
\hline$v^{2} v^{r} w^{m} r^{2}$ & 1 & 0,20 & $85 \%$ & 8 \\
\hline 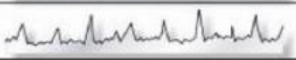 & 1 & 0,20 & $20 \%$ & \\
\hline 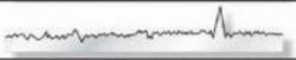 & 1 & 0,08 & $7 \%$ & \\
\hline WNWW & 1 & 0,20 & $25 \%$ & $\Gamma$ \\
\hline WWWWWWW & 1 & 0,30 & $38 \%$ & \\
\hline
\end{tabular}

Fig. 13. Differences between the roughness profile and shape of the Abbott curve [12]

The reduced height of the depression Rvk characterizes the proportion of the dimension of the depression below the core of the profile, reduced by the calculation of the influence of isolated large depressions. The size of Rvk can be significant, e.g. for lubricating contact surfaces (i.e. for keeping the lubricant at the point of contact of the surfaces). For example, for cast iron cylinder liners, a surface finish with large Rvk values has proven to be suitable. [12]

\section{Conclusion}

We define texture, or in other words the structure of a surface, as an area with a huge set of grains, which are located between the surroundings and the surface of the material, i.e. the fixed part. The surface is an extremely complex system where there are repeated (periodic) or random (aperiodic) deviations from the ideal geometric shape of the surface. These deviations can be expressed in several ways, the oldest of which is the line profile, the most common nowadays is probably the expression of the three-dimensional topography of the surface. According to the size of the pitch of the respective irregularities, the surface structure is divided into three components. The basic profile determines the component with the largest pitch of inequalities and is called the P-profile. The component with the smallest pitch is determined by the surface roughness, which in turn is referred to as the R-profile. The component between the largest and smallest spacing is called the waviness of the surface, the $\mathrm{W}$-profile.

The composition of these components on the surface generally directly affects the functional properties of the surface. The often expressed view of the surface - "the smoother the better" - may not always be correct, as there are other factors, e.g. depressions must be left on the surface to hold the lubricating medium to ensure lubrication. In all cases, it is necessary to assess the economic side of things - the increase in the cost of producing a very "smooth" surface may not always correspond to the functional benefit. Therefore, functional use is decisive for the required surface properties. Surfaces that will move against each other will have different requirements from those for surfaces forming sealing surfaces or for surfaces intended for subsequent painting, and so on. It follows from this that measuring and evaluating the surface structure is very important from a practical point of view. 
Much has been written and said about assessing the integrity of a surface. However, most of these articles and professional publications focus on the evaluation of the surface of periodic surfaces, which is logical due to their representation in technical practice. However, the aim of this article is to point out the difference between measuring the surface roughness of periodic surfaces (the technical practice for the most common surfaces) and non-periodic surfaces. Each section of the article focuses on a description of the surface roughness measurement systems for contact and noncontact measurement of non-periodic surfaces and tries to lead the reader to think about the accuracy of the surface measurement procedure. Furthermore, several surface roughness parameters are highlighted in the article, which are suitable for the evaluation of aperiodic surfaces regardless of their subsequent use.

\section{Acknowledgments}

The paper was created with the support of the Motivation System of the University of West Bohemia in Pilsen, part of POSTDOC. And it was co-financed by project GA UWB in Pilsen: SGS-2019-008 "Research and development for innovation in the field of manufacturing processes - Technology of metal cutting III".

\section{Literature}

[1] Thakur, A.; \& Gangopadhyay, S.; (2016) State-of-the-art in surface integrity in machining of nickel-based super alloys, International Journal of Machine Tools and Manufacture, Volume 100, Pages 25-54, ISSN 0890-6955, https://doi.org/10.1016/j.ijmachtools.2015.10.001.

[2] Sogorovic, D.; \& Knezevic, A. (2019). Testing of the Surface Roughness Parameters of Aluminium-Lithium Parts Machined by Milling, Proceedings of the 30th DAAAM International Symposium, pp.0148-0154, B. Katalinic (Ed.), Published by DAAAM International, ISBN 978-3-902734-22-8, ISSN 1726-9679, Vienna, Austria DOI: 10.2507/30th.daaam.proceedings.019

[3] Cekic, A.; Muhamedagic, K.; Cohodar, M.; Begic-Hajdarevic, D. \& Biogradlija, S. (2019). Experimental Investigation of Effect of Overhang Tool Length on Tool Vibration and Surface Roughness, Proceedings of the 30th DAAAM International Symposium, pp.0184-0191, B. Katalinic (Ed.), Published by DAAAM International, ISBN 978-3-902734-22-8, ISSN 1726-9679, Vienna, Austria DOI: 10.2507/30th.daaam.proceedings.024

[4] Kubatova, D. \& Melichar, M. (2019). Roughness Evaluation Using Abbott-Firestone Curve Parameters, Proceedings of the 30th DAAAM International Symposium, pp.0467-0475, B. Katalinic (Ed.), Published by DAAAM International, ISBN 978-3-902734-22-8, ISSN 1726-9679, Vienna, Austria DOI: 10.2507/30th.daaam.proceedings.063

[5] Hronek, O. \& Zetek, M. (2017). The Influences of Cutting Edge Radius on Surface Roughness when Milling Nickel Alloy, Proceedings of the 28th DAAAM International Symposium, pp.1037-1043, B. Katalinic (Ed.), Published by DAAAM International, ISBN 978-3-902734-11-2, ISSN 1726-9679, Vienna, Austria DOI: 10.2507/28th.daaam.proceedings. 144

[6] CHEN, S.; FENG, Ch. \& ZHANG Y. (2018) Surface roughness measurement method based on multi-parameter modeling learning. Measurement [online]., 129, 664-676 [cit. 2020-09-09]. DOI:10.1016/j.measurement. 2018.07.071. ISSN 02632241.

[7] Purkait, B.; Majumdar B. \& Mazumder, R. (2017) Bed roughness and grain sorting- an experimental study over fine to medium sand beds, International Journal of Sediment Research, Volume 32, Issue 3, Pages 384-400, ISSN 10016279

[8] SÝKOROVÁ, J. (2020). Evaluation of surface roughness after machining . Ostrava, 2020. Diploma thesis. VŠB Technical University of Ostrava, Thesis supervisor Ing. et Ing. Mgr. Jan Petrů, Ph.D.

[9] Roughness measurement [online]. [cit. 2020-09-09]. Available from: https://www.mbcalibr.cz/merenidrsnosti.html

[10] ŠTEFAN, V. (2014). Data filtering when measuring surface roughness. Diploma thesis; University of West Bohemia in Pilsen; Thesis supervisor Ing. Zdeněk Pospěch, Ph.D.

[11] Roughness measurement system - roughness parameters [online]. [cit. 2020-09-09]. Dostupné z: https://www.jenoptik.cz/files/094_14-hommel-plakat-drsnost-1000x700mm.pdf

[12] NOVÁK, Z. (2004) Improving the quality of surface structure evaluation. MM Industrial Spectrum. (041213), 84. ISSN 1212-2572. 